\title{
ENLARGED IONIZATION REGION AND WILEY-MCLAREN TYPE GEOMETRY
}

\author{
Ş. Şentürk \\ Dumlupınar University, Department of Physics, Kütahya-Türkiye \\ sukru_sen_2000@yahoo.com
}

\begin{abstract}
The ionization region of the ToF mass spectrometer having the WileyMcLaren geometry was further enlarged on the basis of the Milani-de Heer criteria. The enlarging limits the use of the spectrometer to small cluster range as a result of mass resolution decrease at great deal amount. However the spectrometer is promising for the electron-cluster interaction studies.
\end{abstract}

Key words- Enlarged ionization region, Time of Flight Mass Spectrometer, Metallic clusters, Mass resolution

\section{INTRODUCTION}

Among the various types of time of flight (ToF) mass spectrometer the one with the largest ionization region is most convenient for cluster study. This is because such spectrometer provides more effective ionization region, and therefore able to achieve a strong ion signal without causing fragmentation resulting in better signal to noise ratio. On the other hand for spectrometers with low ionization regions to achieve better signal to noise ratio, the intensity of the ionising beam needs to be increased. This result in problems associated with fragmentation unless one considers fragmentation process of the clusters. The effective ionization scheme is therefore in need of improvement in the signal to noise ratio for the electron-cluster interaction studies. The reported signal to noise ratio via the beam depletion method is poor [1]. Weak signal level encounters mainly due to the low cluster number density.

Large ionization volume spectrometer introduced to cluster field by W.A.de Heer and P. Milani had geometry similar to the Wiley-McLaren type spectrometer with an intermediate field free-region included to the ionization-acceleration zone $[2,3]$. In the original Wiley-McLaren spectrometer, there was no compensation for the effect of an initial velocity of the ions. The ionization range of the spectrometer was around $10 \mathrm{~cm}$. With this system a mass resolution higher than 1000 was obtained. The mass resolution of ToF /MS with large ionisation region was further improved by Chandezon et al [4]. They reported mass resolution of 2000 for an ionization volume of about $5.20 \mathrm{~cm}$. In their study, Milani-de Heer's criteria was used while keeping the Wiley and McLaren geometry i.e. one grid less and hence higher transmission. Performance of both spectrometer were reported for the positively charged cluster $[2,4]$ 
In this study, the ionization region of the ToF - mass spectrometer having the WileyMcLaren geometry was further enlarged implementing the de Heer-Milani-criteria and the resolution determined. The spectrometer performance is presented through negatively and positively charged clusters. The primary focus was the behavior of the mass resolution and whether this approach can be used in the electron-clusters interaction studies. A description of the spectrometer is given below.

\section{DESCRIPTION OF THE SPECTROMETER}

The spectrometer has two distinct parts namely ionization-acceleration zone and the free flight zone. In this arrangement the cluster source is placed along the spectrometer axis at $6^{\circ}$ as shown in figure 1 . The $6^{\circ}$ provide separation of the charged clusters from the neutral one that in turn eliminates possible collisions of ions with the neutral beam.

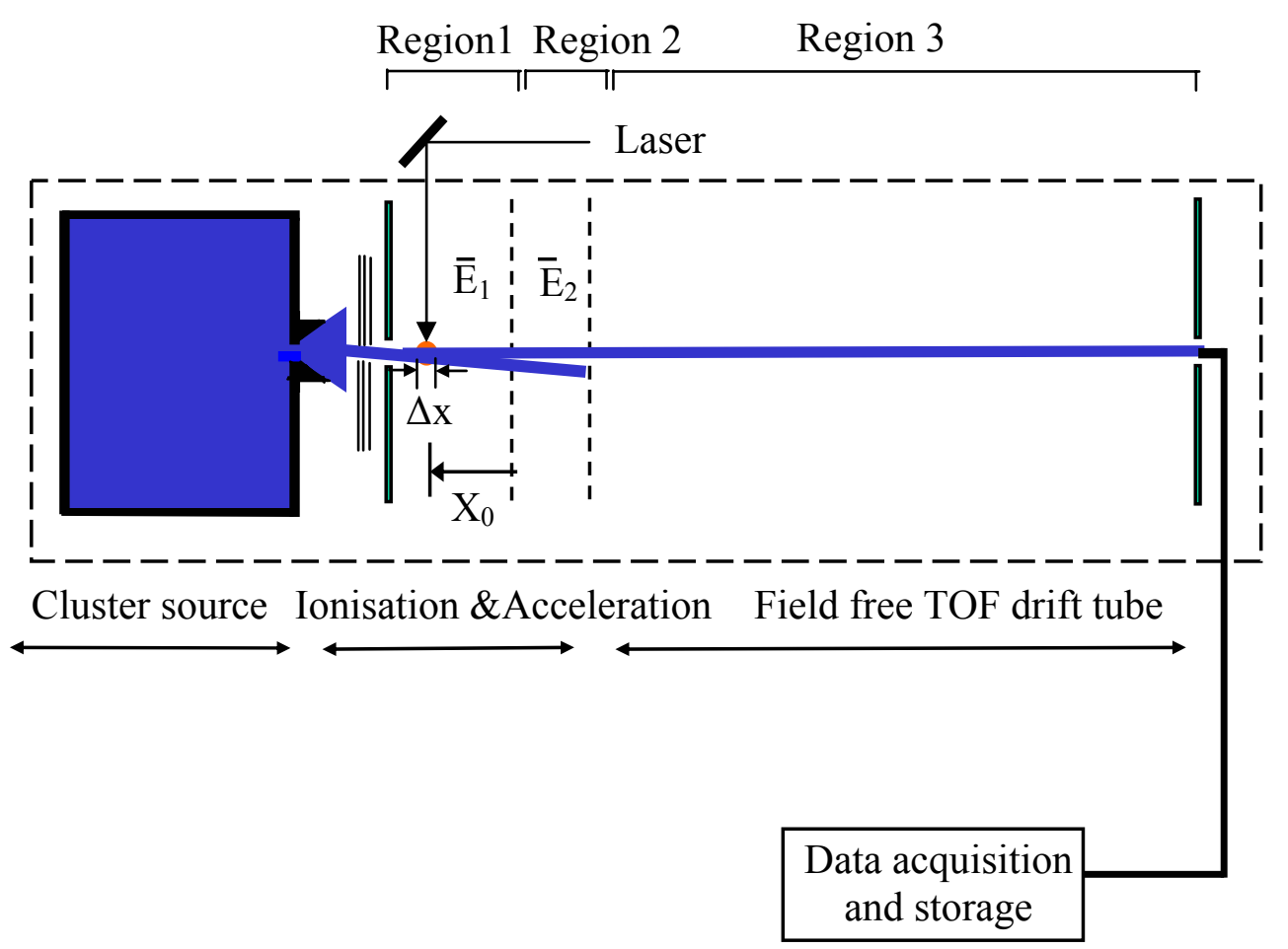

Figure 1: Block diagram of the ToF spectrometer with cluster source.

The ionisation-acceleration zone consists of regions 1 and 2, which are $13 \mathrm{~cm}$ and $3 \mathrm{~cm}$ long respectively. In region 1 the particles are ionised and they are pushed through region 2 by the electric field $E_{1}$. The particles are further accelerated by the electric field $\mathrm{E}_{2}$. A $2.1 \mathrm{~m}$ long free-flight zone follows the ionisation-acceleration zone. In that region the accelerated particles drift freely and they experience no electric field along where the ion packets of different masses are separated. The mass separated particles are 
detected via microchannel plates (MCP detector), from where they pass through the PC for data collection.

\section{THE MILANI-de HEER CRITERIA AND DESIGN OF SPECTROMETER}

The total flight time of a particle having mass $m$ with a charge $q$, starting from the initial distance $\mathrm{x}$ of the first grid and with an initial velocity $\mathrm{v}$ along the spectrometer axis, will be the sum of three partial flight times (these are flight times in each region). For the formalision of the flight time the reduced parameter approach used by Chandezon et al. [4] was kept to simplify the calculations. The reduced initial position of the particle is $\mathrm{X}=\mathrm{x} / \mathrm{L}$, and $\mathrm{S}=\mathrm{v}(\mathrm{m} / 2 \mathrm{qV})^{1 / 2}$, its reduced initial velocity component along the spectrometer axis. Other reduced parameters are $\mathrm{c}$ and $\mathrm{E}$ for the spectrometer, the reduced length and reduced field. The lengths are normalised to $\mathrm{L}$ and the voltages to $\mathrm{V}$. Hence $\mathrm{c}=\mathrm{C} / \mathrm{L}$ is the reduced length of region 2 and $\mathrm{E}=\left(\mathrm{V}_{\mathrm{a}} / \mathrm{V}\right)(\mathrm{L} / \mathrm{A})$ is the reduced value of the electric field in region 1 .

The total flight time via the reduced parameters can be written as

$$
T(x, v)=L \sqrt{m / 2 q V} f(X, S)
$$

where $\mathrm{f}(\mathrm{X}, \mathrm{S})$ is a dimensionless function, and it is given by

$$
f(X, S)=\left[\frac{2\left(\sqrt{S^{2}+E X}-S\right.}{E}\right]+\left[2 c\left(\sqrt{S^{2}+E X+1}-\sqrt{S^{2}+E X}\right]+\left[\frac{1}{\left[\left(\sqrt{S^{2}+E X+1}\right]\right.}\right]\right.
$$

On the basis of criteria, the parameter values of the $f(X, S)$ function determine the property of the spectrometer. The basic idea is to select the parameters of the $f(X, S)$ function that possesses a maximum and a minimum with the initial ion position $\mathrm{X}$ from the first grid between them. In order to meet this requirement the parameter values were selected. The $\mathrm{F}(\mathrm{X}, \mathrm{S})$ plot for selected parameters is given in figure 2 .

This shows a region $\left[\mathrm{X}_{1}, \mathrm{X}_{2}\right]$ where the values of the function are limited between $\mathrm{f}\left(\mathrm{X}_{\min }, \mathrm{S}\right)$ and $\mathrm{f}\left(\mathrm{X}_{\max }, \mathrm{S}\right)$. The region $\left[\mathrm{X}_{1}, \mathrm{X}_{2}\right]$ is an usable ionization region according to the criteria which is around $90 \mathrm{~cm}$. This allows the use of ionization region length of 13 $\mathrm{cm}$ fully that can be reduced to the ionization range value through the applied voltages. However, we tried otherwise to obtain the possible largest range and observe the mass resolution behavior. In this region, the resolution has the value;

$$
\frac{m}{\partial m}=\frac{1}{2} \frac{T}{\partial T}=\frac{1}{2} \frac{f_{\text {mean }}}{f\left(X_{\max }, S\right)-f\left(X_{\min }, S\right)}
$$

where the $f_{\text {mean }}$ is as following 
$f_{\text {mean }}=\frac{f\left(X_{\min }, S\right)+f\left(X_{\max }, S\right)}{2}$

Using the values of the graph given in the figure 2 the resolution for the present arrangement can be determined as 20 . The value is extremely low compared to the reported ionization range. This is due to the enlargement of the ionization region and dependence of the initial velocity component of the cluster. The low mass resolution obviously limits use of the present arrangement. However notice that all effort was for the having larger ionization region.

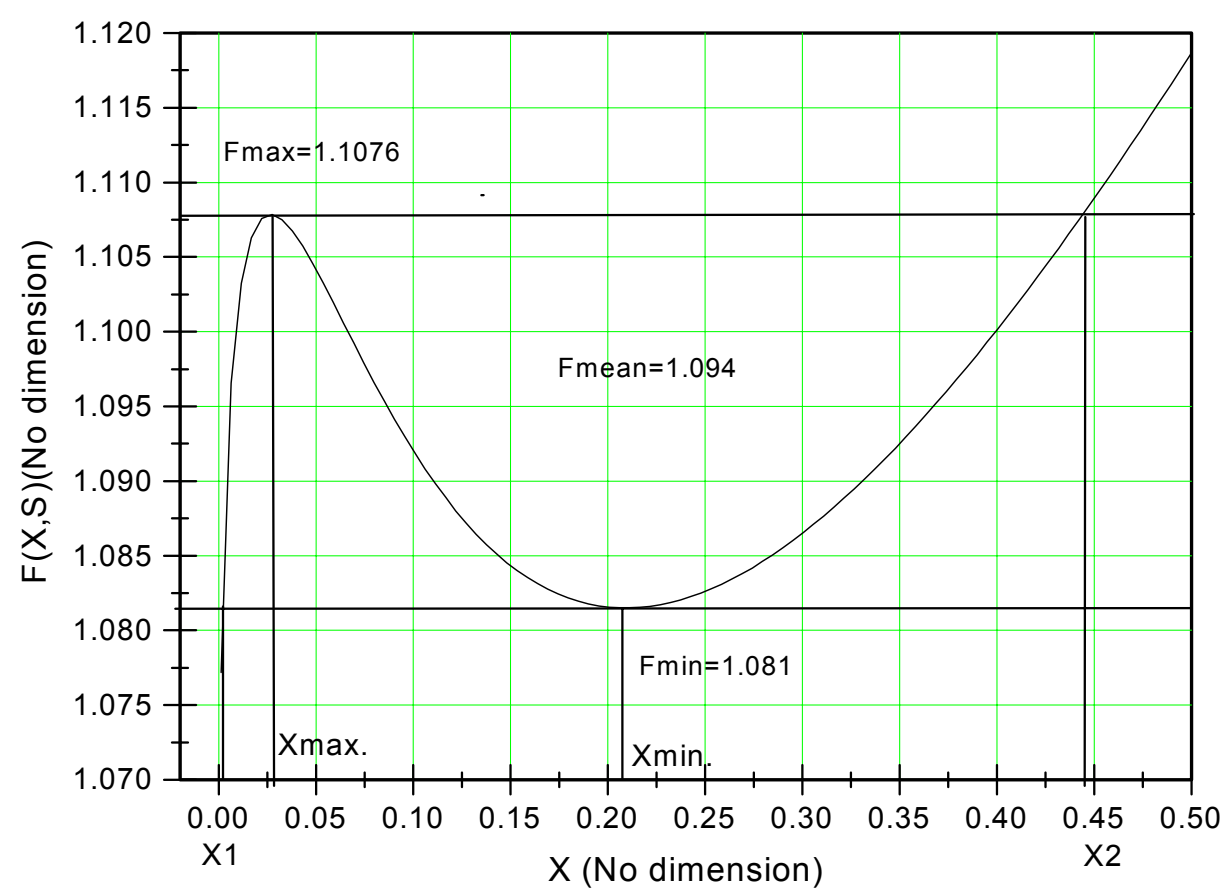

Figure 2: Plot of $\mathrm{F}(\mathrm{X}, \mathrm{S})$ function vs $\mathrm{X}$ initial ion position.

\section{PERFORMANCE OF THE SPECTROMETER}

An obtained mass spectrum for the positively charged potassium clusters containing up to 8 atoms is shown in figure 3 . The mass resolution is very low, however the spectrum illustrates that the ion peaks are separate enough from each other, therefore this resolution would not be a problem for the small cluster range. For detecting the ions, a stable CW high voltage supply was used for the acceleration. The applied voltages are $4.5 \mathrm{kV}$ for $\mathrm{V}_{\mathrm{a}}$ and $6 \mathrm{kV}$ for $\mathrm{V}$. The laser focussing area is around $0.25 \mathrm{~cm}^{2}$ and the distance of focusing position is around $11.5 \mathrm{~cm}$ from the first grid. The MCP's are run at normal mode and then passed through to PC for data processing. The flight times predicted for the potassium clusters, up to 8 atoms, through the equation (1) is shown in Figure 4. The estimated and measured flight times are fairly in agreement. The 
difference between them is probably due to the velocity distribution and to a voltage shift on the acceleration plate supplies.

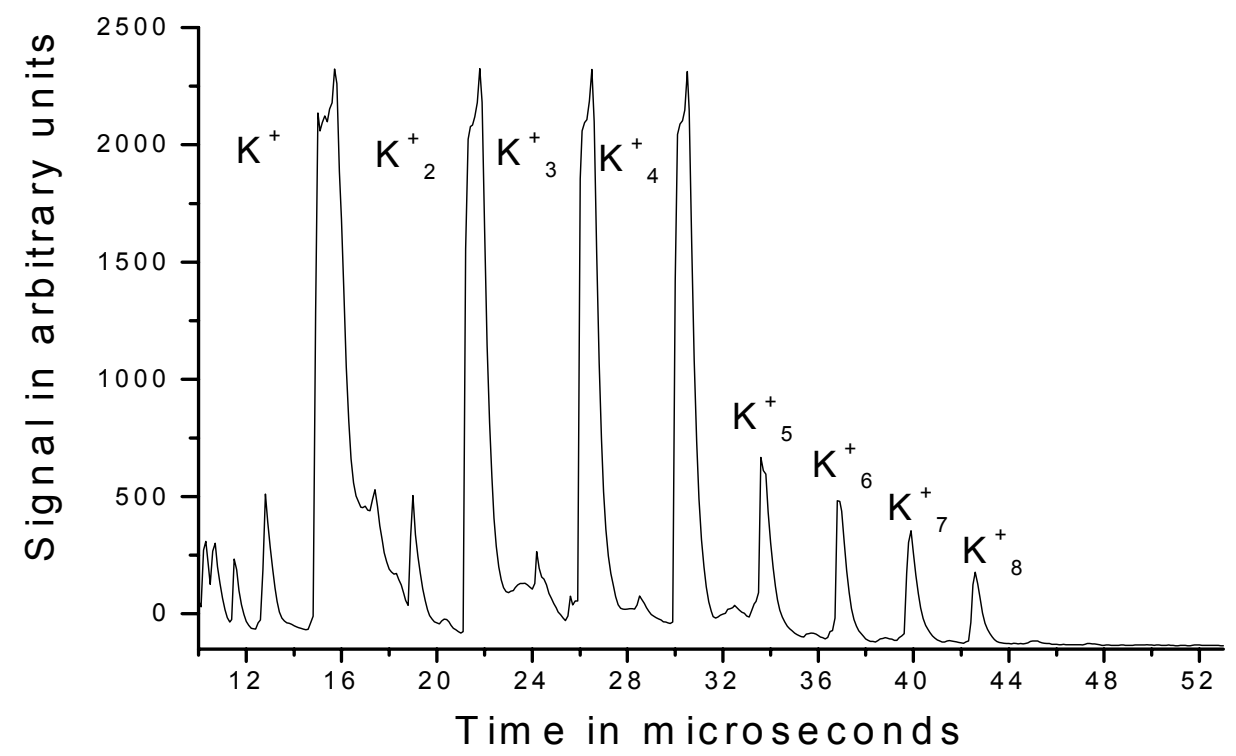

Figure 3: Mass spectrum of the positively charged clusters obtained

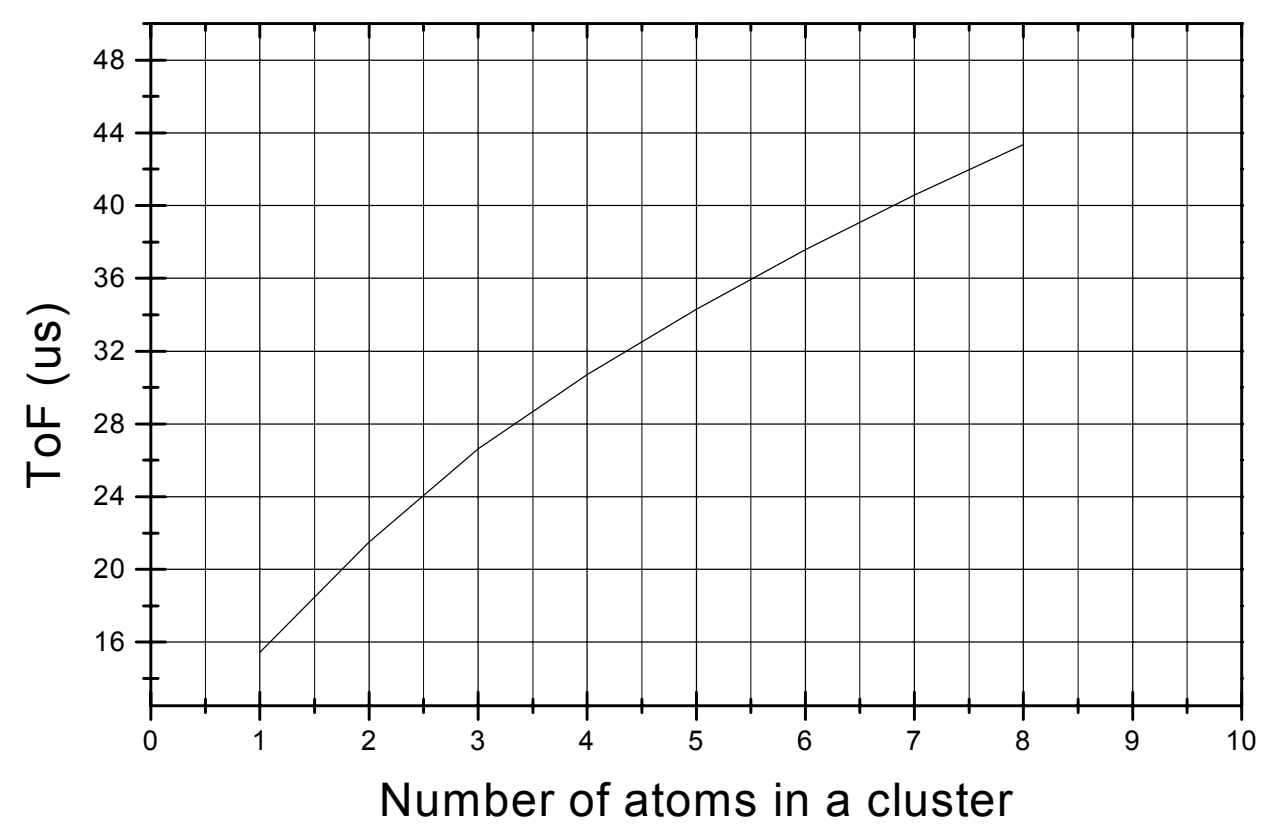

Figure 4: Calculated flight times for the potassium clusters up to 8 . 
Although a mass spectrum for the negatively charged clusters could not be produced as such, the inelastic cross section for them is determined. The agreement of the experimental data with the theoretical prediction indicates the reliability of the obtained data [5]. In the light of this agreement we could say that the enlargement of the ionization region approach can be used for the electron-clusters interaction.

The difficulty associated with the electron interaction experiment is our inability to produce the mass spectrum. Therefore the concern is the flight times of the negative ions. Since the flight times of the positively charged clusters is known the arrival time of the negative ions should not change providing that the same values are kept for the parameters during the set up. The calculation carried out to predict the flight times showed that there is a slight difference between the negative ions and the observed mass spectrum of the positive ions. This is due to the ionization region enlargement. The difference, although is small, was taken into account when the counter electronic was set up. In this way the mass spectrum is fully corrected. Negative pulsar supply was used for the acceleration of negative ions. The time window of the pulses applied to the plates is around $220 \mu \mathrm{s}$. This time window is sufficient for clusters to reach the detector and the rise time of the pulses is $6 \mathrm{~ns}$. In this part of the experiment particular attention needs to be paid to controlling the applied voltages.

\section{CONCLUSION}

Enlarging the ionization region further gives rise to the low mass resolution. This ends up with a result that the spectrometer can be useable for the small cluster range. Although the use of the spectrometer is limited to small cluster range the agreement of the determined inelastic cross section for the small potassium clusters with theoretical prediction suggests that the spectrometer can be applicable in the low energy electroncluster interaction studies. For the higher cluster range, the low mass resolution can cause difficulties. Accordingly one needs to consider the priorities for using the enlarging ionization method.

\section{REFERENCES}

[1] V. Kasperovich, G. Tikhonov, K. Wong, P. Brockhaus and V. V. Kresin, Polarization forces in collisions between low-energy electrons and sodium clusters, Phys. Rev.A 60, 3071-3075, 1999.

[2] Walt A. de Heer and P. Milani, Large ion volume time-of-flight mass spectrometer with position and velocity-sensitive detection capabilities for cluster beams, Rev. Sci.Instrum. 62, 670-677, 1991.

[3] W.C. Wiley and I. H. McLaren, Time of flight mass spectrometer with improved resolution, Rev. Sci. Instrum. 26, 1150-1157, 1955.

[4] F. Chandezon, B. Huber and C. Ristori, A new-regime Wiley-McLaren time-offlight mass spectrometer, Rev. Sci. Instrum. 65, 3344-3353, 1994.

[5] Ş. Şentürk, J. P. Connerade, D.D. Burges and N. J. Mason, Enhanced electron capture by metallic clusters, J. Phys B: At. Mol. Opt. Phys. 33, 2763- 2774, 2000. 